\title{
A genome-wide assessment of genetic diversity and population structure of Korean native cattle breeds
}

\author{
Aditi Sharma', Seung-Hwan Lee ${ }^{2 \dagger}$, Dajeong Lim ${ }^{1}$, Han-Ha Chai ${ }^{1}$ Bong-Hwan Choi ${ }^{1}$ and Yongmin Cho ${ }^{1 *+}$
}

\begin{abstract}
Background: The native cattle breeds are an important genetic resource for meat and milk production throughout Asia. In Asia cattle were domesticated around 10,000 years ago and in Korea cattle are being raised since 2000 B.C. There are three native breeds of cattle in Korea viz. Brown Hanwoo, Brindle Hanwoo and Jeju Black. While one of these breeds, Brown Hanwoo, is a part of a Food and Agricultural Organization and national genetic evaluation plans, others get little attention. This study is an effort to understand and provide a detailed insight into the population structure and genetic variability of the Korean cattle breeds along with other Asian breeds using various methods. In this study we report the genetic variation and structure of the Korean cattle breeds and their comparison with five other Asian cattle breeds along with a panel of animals from European taurine, African taurine and indicine cattle breeds.

Results: Asian cattle were found to be least differentiated which reflects their recent history. Amongst the Asian breeds Hainan, which is an indicine breed, had the lowest gene diversity while Yanbian had the highest followed by Mongolian and Korean cattle. Amongst the Korean breeds Brown Hanwoo had the highest diversity followed by Brindle Hanwoo and Jeju Black. The genetic diversity in Asian cattle breeds was found to be comparable to the European taurines and more than the African taurines and Zebu cattle. Korean cattle breed, Brown Hanwoo was consistently found to be closer to Yanbian, a Chinese cattle breed. We found low divergence and moderate levels of genetic diversity among the native Korean breeds. Indicine introgression from Hainan was seen in other Asian breeds. From Europe, Limousin, Holstein and Hereford introgression was found in Asian breeds.
\end{abstract}

Conclusions: In this study we provide a genome-wide insight into the genetic history of the native cattle breeds of Korea. The outcomes of this study will help in prioritization and designing of the conservation plans.

Keywords: Hanwoo, Cattle, Korea, Genetic diversity, Illumina bovinesnp50 beadchip

\section{Background}

Cattle is known to be domesticated approximately 10,000 years ago near present day Turkey and Pakistan however, the earliest remains of domestic cattle found in north-east Asia dates back to only 5000 years [1]. There were multiple independent domestication events for the world cattle population as suggested by McTavish et al. [2]. Two main established splits of cattle i.e., taurine cattle

\footnotetext{
* Correspondence: variance@korea.kr

${ }^{\dagger}$ Equal contributors

'Division of Animal Genetics and Bioinformatics, National Institute of Animal Science, RDA, 1500, Kongjwipatjwi-ro, Iseo-myeon, Wanju-gun, Jeollabuk-do 55365, Republic of Korea

Full list of author information is available at the end of the article
}

(hump less) and indicine cattle (humped) are considered to have descended from aurochs which are the wild ancestors of the present day cattle breeds. Modern day breeds are a result of natural and artificial selection and adaptation to the local climate. Due to various reasons about $16 \%$ of the cattle breeds are already extinct and 30\% face the risk of extinction. Preserving the local cattle breeds thus becomes necessary in order to prevent the depletion of the genetic resources of the country. Accessing the genetic diversity of a population provides an insight into the history, genetic structure and current status of the population which form the very basis for genetic improvement. 
In North-East Asia (China, Mongolia, Korea and Japan), most of the cattle breeds are taurine type but China also has some indicine/zebu type and hybrid breeds (Table 1). Asian cattle are different from other taurine type cattle as they have been reported to have an independent mitochondrial origin [3]. Asian cattle are known to have migrated from North China to Korea via Mongolia and from Korea to Japan. Also, Korean cattle are believed to have descended as a crossbreed from European Bos primigenius and Indian Bos indicus. In Korea there are three native taurine type cattle breeds viz., Korean Brown cattle (BH, Hanwoo), Korean Brindle cattle (BNH, Chikso) and Jeju Black (JB, Jeju Heugu). These breeds differ from each other in the coat color and levels of nose darkness [4]. Geographically, these breeds are quite distantly located. Hanwoo is a mainland breed while Brindle Hanwoo and Jeju Black are the island breeds. All the three breeds are well adapted to the local climatic conditions. While Hanwoo can withstand temperatures as low as -20 , Jeju Black shows remarkable adaptation to hot and humid climate of Jeju Island. The prevalence of brown Hanwoo is more abundant ( 3 million animals) as compared to the other breeds as it is the only one recognized and registered by the government for selection and breeding programs. Also, Hanwoo is believed to share its ancestor

Table 1 Breeds, breed code and number of samples used in the study

\begin{tabular}{lllll}
\hline Breed Name & Breed Code & Sample Size & Type & Meat/Dairy \\
\hline Brown Hanwoo & BH & 120 & Taurine & Meat \\
Brindle Hanwoo & BNH & 20 & Taurine & Meat \\
Jeju Black & JB & 20 & Taurine & Meat \\
Yanbian & YB & 39 & Taurine & Meat/Draught \\
Wagyu & WAGY & 12 & Taurine & Meat \\
Qinchuan & QC & 4 & Hybrid & Meat \\
Hainan & HN & 4 & Indicine & Draught \\
Luxi & LX & 5 & Hybrid & Meat \\
Mongolian & MG & 5 & Taurine & Meat \\
Angus & ANG & 24 & Taurine & Meat \\
Brahman & BRM & 25 & Indicine & Meat \\
Nelore & NEL & 21 & Indicine & Meat \\
Gir & GIR & 24 & Indicine & Dairy \\
Brown Swiss & BS & 22 & Taurine & Dairy \\
Hereford & HFD & 21 & Taurine & Meat \\
Holstein & HOL & 60 & Taurine & Dairy \\
Jersey & JER & 49 & Taurine Dairy \\
Limousin & LMS & 25 & Taurine Meat/Milk \\
N'Dama & ND & 56 & Taurine & Meat/Milk \\
Sheko & SHK & 20 & Taurine & Meat/Draught \\
\hline
\end{tabular}

with Yanbian, a Chinese cattle breed, until the last century [5]. Like most other Asian cattle breeds the history of Korean cattle breeds too is not very well documented. There are some references of these animals to be used as draft animals and also used in religious sacrifices [6]. It was in 1979 that a government regulated breeding program called "Hanwoo-Gaeryang-Danji (HGD)" was initiated for the native Hanwoo cattle [7]. Hanwoo is mainly selected for the carcass weight and meat quality traits like Marbling, backfat thickness, Loin eye area. Owing to the government led programs Hanwoo is now one of the superior commercial livestock breeds of Korea. Recently, government has shown an increased interest towards the conservation of other breeds and also towards the development of these breeds as an alternate beef breed. Availability and cost of genome-wide Single Nucleotide Polymorphism (SNP) panel had made it a popular choice of method to accesses diversity of various livestock species $[8,9]$. In this study we utilized illumina BovineSNP50 BeadChip Ver. 1 (Illumina, San Diego, CA, USA) to genotype three native Korean cattle breeds along with Yanbian resulting in 54,609 genotyped SNPs. We combined data from 11 representative breeds from the BovineHapmap dataset comprising of European taurine, African taurine and Indicine (Zebu) cattle and 5 Asian breeds from data published by Decker et al. [10].

In our study we present a detailed insight into the genetic diversity and structure of Korean cattle breeds in comparison with Chinese, Mongolian and Japanese breeds. The outcomes of this study would shed light on the genomic structure of Korean breeds as well as other Asian breeds and this information could be used as a primer to design conservation strategies and breeding programs.

\section{Methods \\ Animals and genotyping}

Blood samples were collected from Brown Hanwoo (BH), Brindle Hanwoo (BNH) and Jeju Black (JB). All three breeds are found in their native track in three different regions of the country. Breeds and number of samples used for the study are described in detail in Table 1 . BH is a mainland breed while $\mathrm{BNH}$ and JB are island breeds. Utmost care was taken to avoid any crossbreds during sampling. YB (Chinese cattle) samples were made available by Dr. Lee SH from Chungnam National University in Daejon, South Korea. Genotyping data for the Asian cattle breeds was downloaded from dryad.org [11]. European taurine, African taurine and Zebu data was used from the Bovine Hapmap project. All the genotype data was then merged to make one final dataset. In the final dataset there were 576 samples and 35598 SNPs.

Genomic DNA for genotyping assays was extracted from the blood sample using DNeasy 96 Blood and 
Tissue Kit (Qiagen, Valencia, CA, USA). DNA quantification was performed using a NanoDrop 1000 (Thermo Fisher Scientific Inc., Wilmington, DE, USA). DNA samples were submitted for genotyping with total DNA of $900 \mathrm{ng}, 260 / 280$ ratio $>1.8$, and DNA concentration of $20 \mathrm{ng} / \mathrm{ul}$. The genotyping for animals was done by the Animal Genome \& Bioinformatics Division of the National Institute of Animal Science, RDA, Korea, using a BovineSNP50 BeadChip Ver.1. (Illumina, San Diego, CA, USA).

\section{Quality control of the SNP data}

Genotype data was imputed using Beagle program [12]. Plink version1.09 (http://pngu.mgh.harvard.edu/purcell/ plink/) [13]) was used for the quality control of the raw genotype data. After merging all the genotype data we had a total of 35,598 SNPs. SNP genotypes were subjected to filtering based on Minor Allele Frequency $(\mathrm{MAF})>0$. 001, Hardy Weinberg Equilibrium <1E-06 and genotype frequency (0.05). 10,925 markers were removed based on Hardy Weinberg test, 1664 markers were removed based on genotype rate, and 842 markers were removed based on MAF. After Quality control the final dataset consisted of 22,672 SNPs. A total of 576 samples were analyzed in the study.

\section{Genetic diversity and population differentiation analysis}

To understand the genetic diversity of the cattle populations we used Hierfstat R package [14]. Genetic similarities between breeds were accessed with their pairwise Fst values. The Fst values describes the difference in allele frequencies between two independent populations with a potential value of 0 to 1 , with 1 being the most different/ distantly related. Fst-distance matrix was then used for the hierarchical clustering of the breeds. Poppr $\mathrm{R}$ package [15] was used to calculate the Provesti's absolute genetic distances between populations and further compute a Neighbor joining tree.

\section{Population structure}

Population structure of the Korean cattle breeds was studied using multivariate approach and model based methods. Multi-dimensional scaling (MDS) was used to capture the preliminary glimpse of the genetic structure of the Korean cattle populations and also to remove outliers, if any. MDS (Additional file 1: Figure S1) was computed using Plink version1.09 and plotted in $\mathrm{R}$ version 3.2.2 (R Development Core Team, 2008). Principal component Analysis (PCA) and Discriminant analysis of Principal components (DAPC) was performed for the genetic clustering of individuals and breeds. Adegenet [16] $\mathrm{R}$ package was used for the PCA and DAPC analysis. We retained 100 principal components for DAPC which explained $\sim 52 \%$ of the total variance of the data.
Unsupervised hierarchical clustering was performed using the Admixture 1.23 software [17]. Admixture performs maximum likelihood estimation of individual ancestries from multilocus SNP genotype datasets. An in-house $\mathrm{R}$ script was then used to plot the ancestry of individuals of different breeds. While plotting, individuals were ordered according to the fraction ancestry they shared with other individuals. And as each individual shared a different proportion of ancestry with different individuals of different breeds, not necessarily all the individuals of the same breed grouped together in the final plots.

Patterns of population splits and mixtures in the history of the populations were studied using Treemix [18] program. Allele frequencies in the 20 cattle populations were used to infer the structure.

\section{Results}

\section{Genetic diversity and differentiation}

The genetic diversity within populations was accessed as a measure of heterozygosity i.e., expected heterozygosity (Hs) and observed heterozygosity (Ho). Gene diversity in three Korean cattle breeds was found to be slightly less (0.257) than the European taurine cattle breeds (0.274) and higher than the zebu cattle breeds (0.162). YB cattle breed had $\mathrm{Hs}=0.271$ while the Korean native breeds including $\mathrm{BH}, \mathrm{BNH}$ and JB had Hs value of 0.257 (Table 2). Amongst the Asian breeds $\mathrm{HN}$, which is an indicine breed, had the lowest gene diversity while YB had the highest followed by MG and Korean cattle. Amongst the Korean breeds $\mathrm{BH}$ had the highest diversity followed by $\mathrm{BNH}$ and JB. Fis was used to quantify for the nonrandom mating (Inbreeding) and we found less inbreeding than expected as Fis values were negative for all the breeds ranging from -0.16 in BRM to -0.23 in $\mathrm{HN}$. Among all the three native breeds $\mathrm{BNH}$ had the lowest Fis value. The negative Fis values averaged over all the loci indicated a lack of population structure in the populations. Given the small population size of native Korean breeds $(\mathrm{BNH}=4000$ and $\mathrm{JB}=1000)$ considerable diversity still exists in the native populations.

Genetic differentiation between the 20 populations was studied using pairwise Fst estimates (Additional file 2: Table S1). Genetic differentiation between both $\mathrm{BH}$ and $\mathrm{BNH}$ and $\mathrm{BH}$ and $\mathrm{JB}$ was 0.02 while between $\mathrm{BH}$ and $\mathrm{YB}$, $\mathrm{QC}, \mathrm{LX}, \mathrm{MG}$ it was 0.01 . Amongst the Korean native breeds Fst value between $\mathrm{BNH}$ and JB were the highest (0.06). Korean breeds had the lowest pairwise Fst values compared to other taurine and zebu breeds. Amongst the three native breeds the Fst estimates of $\mathrm{BH}$ with other European taurine breeds were the least. The island breeds $\mathrm{BNH}$ and JB were found to be more differentiated from the taurine and zebu breeds. On an average, the genetic differentiation between the Asian breeds was found to be less than the other breeds in the study. 
Table 2 Genetic diversity in the 20 cattle breeds as measured using $\mathrm{Hs}$, $\mathrm{Ho}$ and Fis

\begin{tabular}{lllll}
\hline S. No & Breed & $\begin{array}{l}\text { Expected } \\
\text { Heterozygosity } \\
(\text { Hs) }\end{array}$ & $\begin{array}{l}\text { Observed } \\
\text { Heterozygosity } \\
(\text { Ho) }\end{array}$ & $\begin{array}{l}\text { Inbreeding } \\
\text { (Fis) }\end{array}$ \\
\hline 1 & Brown Hanwoo & 0.2625 & 0.3352 & -0.2100 \\
2 & Brindle Hanwoo & 0.2551 & 0.3310 & -0.2298 \\
3 & Jeju Black & 0.2546 & 0.3268 & -0.2156 \\
4 & Yanbian & 0.2710 & 0.3437 & -0.2048 \\
5 & Wagyu & 0.2340 & 0.3039 & -0.2203 \\
6 & Qinchuan & 0.2515 & 0.3162 & -0.1999 \\
7 & Hainan & 0.1374 & 0.1795 & -0.2348 \\
8 & Luxi & 0.2156 & 0.2687 & -0.1749 \\
9 & Mongolian & 0.2649 & 0.3317 & -0.1894 \\
10 & Angus & 0.2798 & 0.3611 & -0.2227 \\
11 & Brahman & 0.1764 & 0.2225 & -0.1607 \\
12 & Nelore & 0.1542 & 0.2019 & -0.1990 \\
13 & Gir & 0.1546 & 0.2005 & -0.2297 \\
14 & Brown Swiss & 0.2553 & 0.3342 & -0.2314 \\
15 & Hereford & 0.2930 & 0.3806 & -0.2297 \\
16 & Holstein & 0.2875 & 0.3711 & -0.2286 \\
17 & Jersey & 0.2505 & 0.3234 & -0.2167 \\
18 & Limousin & 0.2801 & 0.3582 & -0.2146 \\
19 & N'Dama & 0.2106 & 0.2721 & -0.2114 \\
20 & Sheko & 0.2087 & 0.2663 & -0.2013 \\
\hline
\end{tabular}

$\mathrm{Hs}$ is the expected heterozygosity; Ho is the observed heterozygosity; Fis is the inbreeding coefficient

Provesti's genetic distances between populations were calculated using adegenet $\mathrm{R}$ package and were plotted as a neighbor joining tree (Additional file 1: Figure S2). The results corroborated well with the Fst analysis. Three distinct groups viz. European taurine, Asian taurine and Zebu were observed. . Korean cattle along with Japanese WAGY and Chinese YB cattle formed a separate group apart from European and African taurines. $\mathrm{BNH}$ and $\mathrm{BH}$ culminated on the same node. Due to geographical proximity $\mathrm{YB}$ is believed to be closely connected to BH until the Korean War [19]. HN, LX, QC, SHK and ND clubbed with zebu cattle while MG formed a group with European taurine cattle. The Korean cattle cluster was found between the European taurine on one side and Zebu on the other. It reflects the influence of European taurine and Zebu cattle on the present day Korean and Japanese cattle breeds. $\mathrm{BH}$ was found to be more closely related to $\mathrm{YB}(0.097)$, followed by $\mathrm{BNH}$ $(0.143)$ and JB (0.142). Amongst the three native breeds, $\mathrm{BNH}$ and JB were found to be most distantly related than others (0.176). Genetic distances of Korean breeds with that of other breeds were the least with LMS (0.22) and the highest with $\mathrm{HN}$ (0.32). Compared to other
Asian breeds genetic distance of BH from WAGY was found to be the smallest $(0.202)$.

\section{Population structure}

Population structure of the Korean cattle breeds was studied using multivariate approach and model based methods. Principal component Analysis (PCA) was used to place the Korean Cattle breeds with respect to the European taurine, African taurine and Zebu. PCA is particularly important as it is a powerful method to capture the variation in the genotypic dataset. In our study PCA grouped the individuals in one cluster depending on the origin of population. We observed twenty clearly separated clusters (Fig. 1). The first component split the data according to taurine/indicine split and the second component divided the data according to African/European taurine split. $\mathrm{BH}, \mathrm{BNH}, \mathrm{JB}$ and $\mathrm{YB}$ individuals formed their individual clusters and due to genetic relatedness the clusters were formed almost overlapping with each other. Along with the closeness of Korean breeds amongst themselves they also showed closeness to WAGY. Other Asian cattle breeds lied in between the zebu and Korean cattle cluster. MG cattle formed a cluster close to Korean and Japanese cattle. In our analysis, Korean breeds formed the most compact cluster compared to other breeds known to share same ancestry. SHK (Ethiopian cattle breed), LX, ND and QC placed themselves center to all the major clusters i.e. zebu, European taurine and African taurine cluster. This shows the influence of European and indicine cattle breeds on the formation of these Asian and African breeds.

We then performed the DAPC analysis. DAPC method transforms the data using PCA and then uses the discriminant analysis to identify the clusters. In our analysis 100 PCs were retained which explained $\sim 52 \%$ variation. Results obtained from the DAPC analysis corroborated with those obtained from PCA analysis. Individuals were correctly assigned to their respective clusters. We initially started this study with a total of 21 populations. The $21^{\text {st }}$ population used in the study was a local Korean cattle breed called "Chosun". But based on DAPC, PCA and MDS results we understood that these animals were only crossbreds so we removed the entire population from the analysis and used only 20 populations in this study (Additional file 1: Figure S3).

\section{Model-based population structure}

We performed unsupervised hierarchal clustering of our data as implemented in Admixture software. Admixture estimates the ancestry in a model-based manner from the autosomal SNP panel from a set of unrelated individuals. The data was then plotted in order of fraction ancestry that they shared with other breeds (Fig. 2). In our study at $\mathrm{K}=3$, we observed separation of European, Zebu and Asian -African taurine cattle breeds. At $\mathrm{K}=3$ 


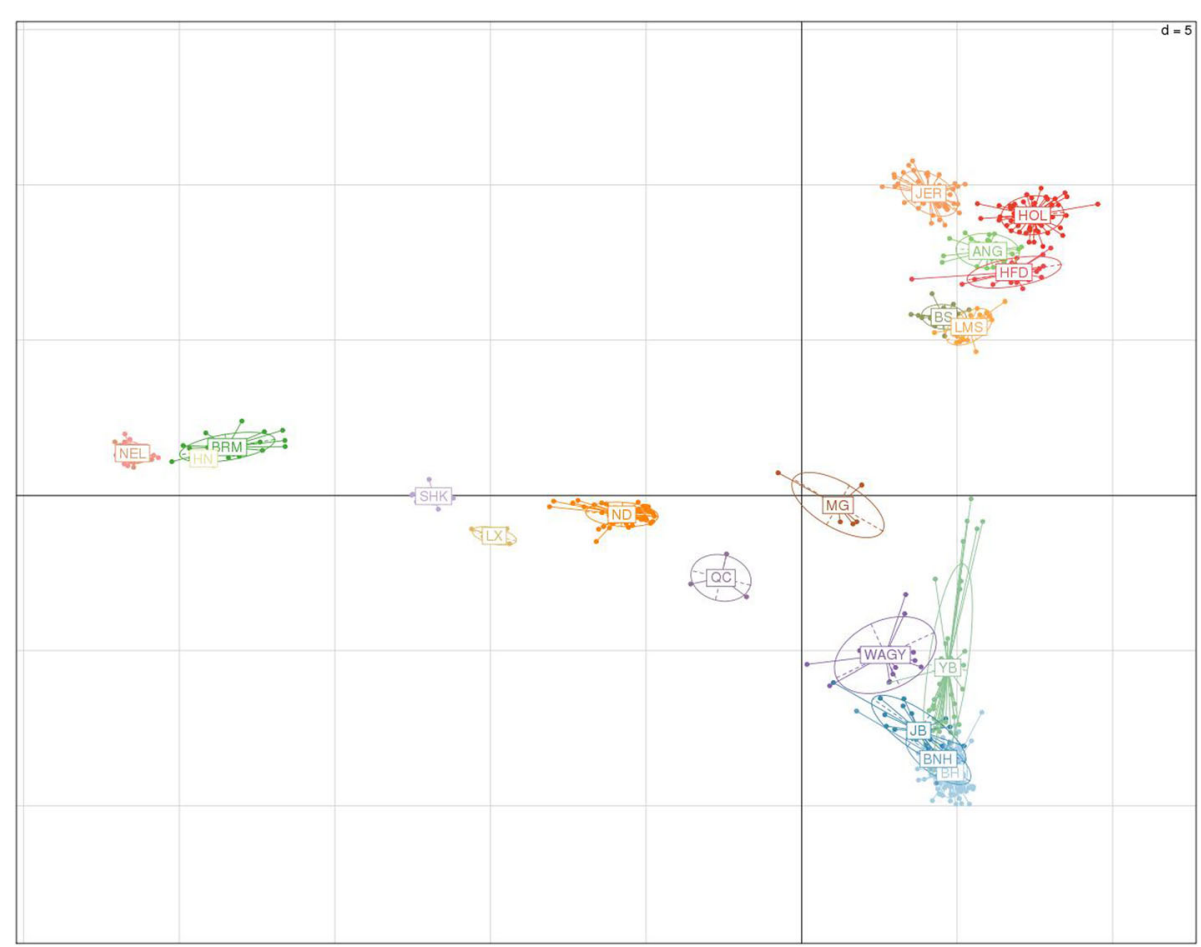

Fig. 1 PCA plot of the 20 cattle breeds plotted using 576 animals and 22,672 SNPs spread across the genome

Asian and African N'Dama cattle formed one group together. Asian cattle breeds formed a separate group from N'Dama at $K=4$. Variable proportion of admixture from Zebu, European and African breeds were observed in Asian breeds. At $\mathrm{K}=4$, most of the $\mathrm{BH}$ animals showed little or no admixture from any other breeds. At $K=6$, LX, which is a hybrid and HN, which is zebu, formed a group with BRM, GIR and NEL. SHK and LX shared $\sim 50-60 \%$ ancestry with zebu breeds. SHK was formed mainly from Zebu and N'Dama but it also showed some admixture with Asian breeds. LX, was formed from Zebu and Asian breeds with some admixture from European and African cattle. Asian breeds didn't differentiate into separate breeds until $K=10$. At $K=10$, WAGY separated as a separate breed while other Asian breeds (Except LX and $\mathrm{HN}$ ) formed one group together. The optimum value of $\mathrm{K}$ was found to be $\mathrm{K}=15$ (Additional file 1: Figure S4) and at this value of $\mathrm{K}$ the Asian breeds started to show differentiation. While some of the individuals of $\mathrm{BH}, \mathrm{BNH}$ and JB made two different groups, most of them still clubbed together. YB individuals formed a group with $\mathrm{BH}$ at almost all values of $\mathrm{K}$. The Asian taurines differentiated well from the African and European taurines, however some European nad African introgression was still seen in the Asian cattle breeds. Along with the European and African admixture Korean breeds showed admixture amongst themselves too. A group of $\mathrm{YB}$ animals were found to share $20 \%$ ancestry with LMS. MG and QC cattle were found to have multiple ancestries. YB shared a large proportion $(\sim 50-60 \%)$ of ancestry with $\mathrm{BH}$ and $\sim 10 \%$ each with $\mathrm{BNH}$ and $\mathrm{JB}$. Both island breeds $\mathrm{BNH}$ and $\mathrm{JB}$ were found to have $\mathrm{BH}$ admixture in them. Admixture of some fraction of African cattle was observed in Asian cattle breeds.

Treemix analysis was used to study the population splits and gene flow (Fig. 1). We first constructed a phylogenetic tree without adding any migration events followed by adding upto 8 migration events for value. Without any migration events we saw all the 20 populations divide into two major groups i.e. Taurine and Indicine. Within taurine, Korean cattle breeds along with Japanese WAGY and Chinese YB formed a separate group. HN and LX formed a group with indicine cattle breeds. As we added migration events we found influence of European cattle, LMS on both Asian and African cattle. When adding more migration evnts than 8 we found introgression from $\mathrm{HOL}$ and HFD into the Asian cattle breeds. Introgression of Indicine genetic component from $\mathrm{HN}$ was seen in the Asian breeds. We also found influence of MG on the Chinese LX and QC breeds.

\section{Discussion}

In our analysis, diversity was assessed as a measure of expected heterozygosity ( $\mathrm{Hs}$ ) and observed heterozygosity (Ho). Diversity in Korean cattle breeds was found to be more than Zebu and African Taurine and less than 


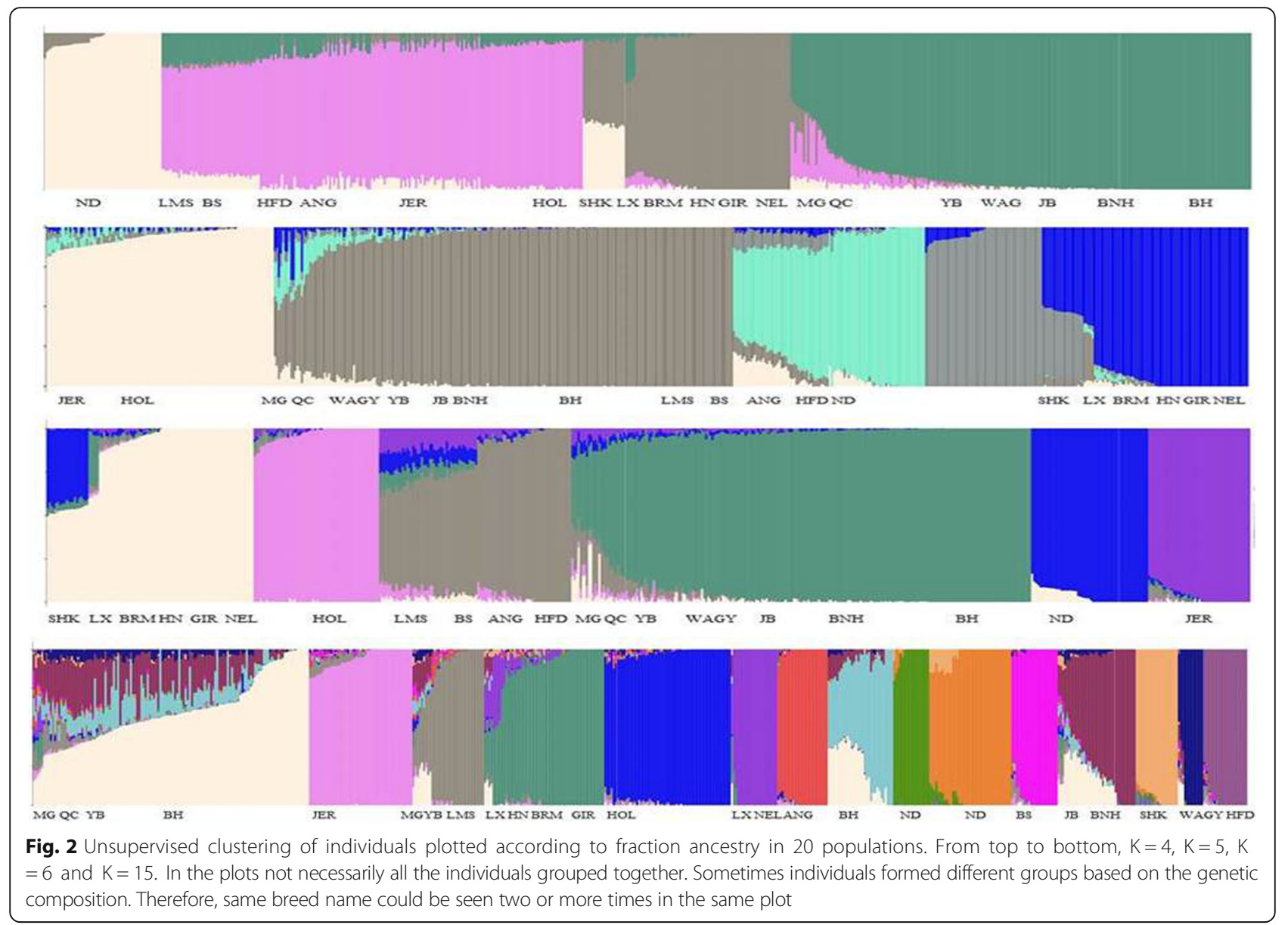

European Taurine. This could be attributed to the recent genetic history of Korean cattle breeds. However, genetic diversity of Korean cattle breeds in our study was considerably lower than that reported by Kim et al. [20]. This might be because Kim et al. used microsatellite markers for the analysis. Also the average observed heterozygosity value for Korean cattle in our study was found to be 0.33 while Edea et al. [9], based on $8 \mathrm{k} \mathrm{Illu-}$ mina SNP chip, reported Ho value to be 0.41 . In both the studies observed heterozygosity value was found to be higher in Korean cattle breeds than the African breeds. The observed heterozygosity values in our study for Korean breeds were similar to that observed by Strucken et al. [21]. Within the Korean breeds observed heterozygosity was found to be considerably higher than the expected heterozygosity. Observed heterozygosity values were found to be least in JB followed by $\mathrm{BNH}$. This could be attributed to their lower population size as reported by Choi et al. [22]. However, despite the small population size the observed heterozygosity values were not remarkably different from the mainland breed $\mathrm{BH}$.
In Korea only $\mathrm{BH}$ has a dedicated breeding program and the number of animals is $\sim 3$ million while number of animals for JB and BNH is only a few thousands. We used Fis as a measure to study inbreeding within these populations. Fis values indicated an excess of heterozygotes in $\mathrm{BNH}(-0.230)$ and $\mathrm{JB}(-0.216)$ which are the island populations. Compared to $\mathrm{BH}(-0.210)$, YB $(-0.205)$ was found to be less inbred. The Fis values in our study were different from that reported by Choi et al. [5]. This could be because of the use of different type of data (microsatellite markers) for the calculations. Korea follows a $20 \mathrm{KPN}$ system in the breeding program. In this program 20 superior bulls are used for artificial insemination across the country. So, despite a good population size of around three million, $\mathrm{BH}$ is found to be more inbred than other Korean populations. Given the population sizes, selection strategies and implementation of designed breeding programs elevated Fis was expected in this domestic cattle breed. Inbreeding in Korean populations on an average was similar to the European taurine cattle breeds. Zebu breeds were found to be least inbred amongst these fifteen populations. 
Pairwise Fst was used to study the population differentiation between the twenty breeds in the study. The Fst values for Korean cattle breeds in our study were lower than the European breeds ranging from 0.02 and 0.06 . $\mathrm{YB}$ and $\mathrm{BH}$ were found to be least differentiated from each other $(\mathrm{Fst}=0.01)$. JB and $\mathrm{BNH}$ were found to be most differentiated from one another $(F s t=0.06)$ while they were found to be less differentiated from $\mathrm{BH}$ which is a mainland breed. The divergence of JB and $\mathrm{BNH}$ in two different directions from the mainland breed makes a good example of the classical island model. Lower Fst values in the Korean populations suggest that they had not yet differentiated well into completely separate/independent breeds. While BH was found to be closer to MG, LX, HN and QC and WAGY (0.02), $\mathrm{BNH}$ and JB were found to be more differentiated from other Asian breeds. Based on Fst values, we found that Asian breeds were still genetically closer to each other than the European, African taurine and Zebu cattle.

We also found evidence of an admixed lineage of Korean cattle breeds. Influence from European taurine and Indicine cattle in varying proportions were observed in the Korean cattle breeds. Genetic influence of Korean cattle breeds from LMS and BS was nicely captured by PCA and DAPC plots. Admixture analysis (Fig. 2) at all the values of $\mathrm{K}$ starting from $\mathrm{K}=3$ showed small proportion of admixture of Korean cattle with African cattle. However, based on treemix analysis (Fig. 3) there was no evidence of direct gene flow between African and Asian breeds. We found the introgression of HN in MG, LX and QC. Gene flow from European cattle breeds LMS, HFD and HOL was also seen in Asian cattle. Based on various population metrics used in the study $\mathrm{BH}$ was consistently found to be closer to YB than the other native breeds. The differences in the breeds could be attributed to the selective breeding of $\mathrm{BH}$. Our study also showed an island effect on the JB and BNH. The genetic diversity in Korean cattle breeds was found comparable to the European taurines.

\section{Conclusion}

Modern day Asian cattle are a result of introgression from various European and Indicine breeds. Mongolian, Qinchuan and Yanbian cattle still contain a high level of admixture from various other breeds while present day Korean cattle were seen to be less admixed. While Korean cattle were found to be less admixed with the breeds outside Korea, they still were found to be admixed amongst themselves. Based on all the population metrics used to study genetic diversity we conclude that the Korean populations are still very closely related and have not yet

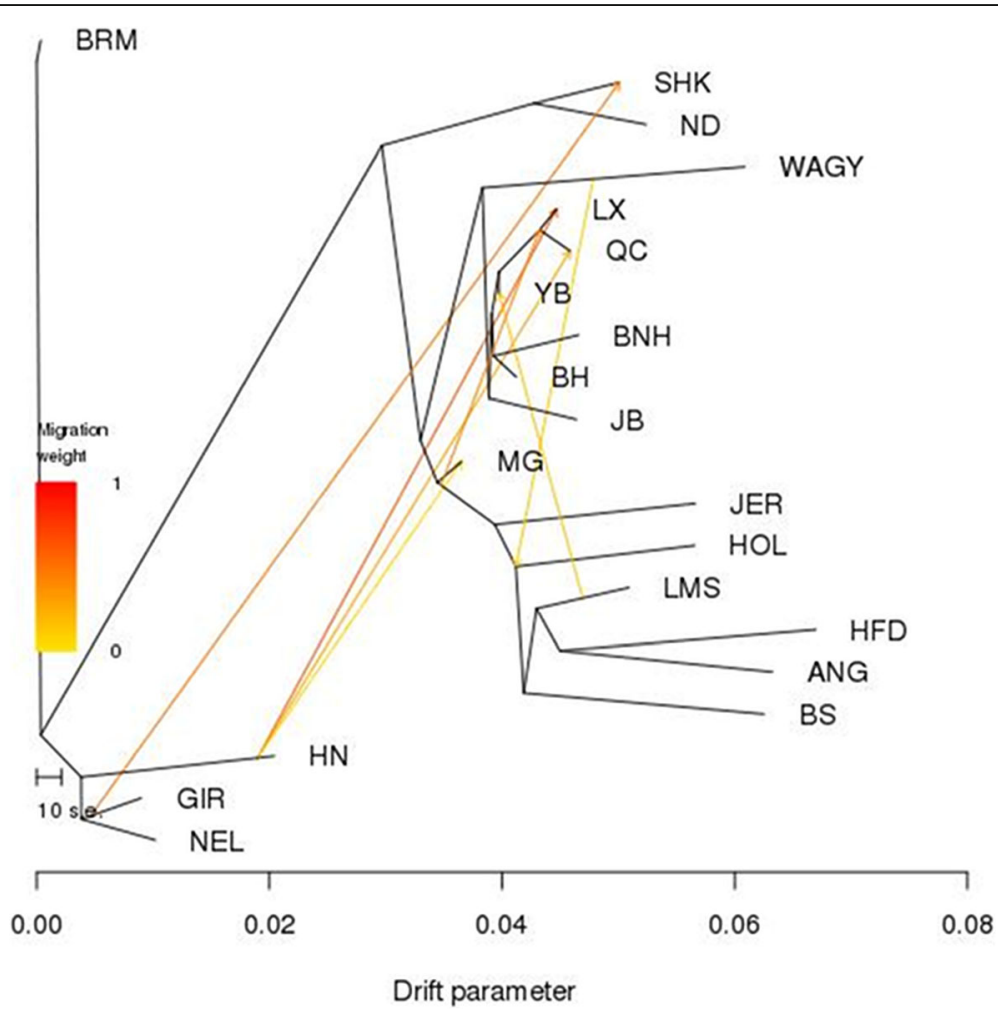

Fig. 3 Maximum likelihood tree inferred from 20 cattle breeds with seven migration edges. Brahman (BRM) cattle breed was used as an outgroup. The scale bar depicts ten times the average standard error of the estimated entries in the sample covariance matrix 
differentiated enough to be considered as separate breeds. These breeds could rather be referred to as the subpopulations of $\mathrm{BH}$. YB was found to be closest to $\mathrm{BH}$ and thus it could be developed as an alternate meat breed of the country. Since YB is an unselected population it can also serve as a model to study the effect of selection and breeding on $\mathrm{BH}$. $\mathrm{BNH}$ and JB are two valuable genetic resources of the country and we suggest relevant measures to be taken to increase the number of individuals in the two island breeds and thus prevent the loss of diversity that may occur due to small population size.

\section{Additional files}

Additional file 1: Figure S1. MDS plot of the 20 breeds. Each color in the plot corresponds to the color shown in the legend. Figure S2: Provesti's genetic distances based Neighbor-Joining tree of 20 cattle breeds. Tree is clearly divided into three groups - one group consists of the Zebu and composite cattle, one consists of European taurine and one group is formed entirely of Asian breeds, mostly Korean. Figure S3. DAPC plot of 4 Korean breeds. This includes Chosun cattle (CS) breed which was later removed from the study. Figure S4: Optimum value of $k$ for the admixture analysis. (DOCX 233 kb)

Additional file 2: Table S1. Pairwise Fst values between 20 populations used in the study. Table S2. Absolute genetics distance or Provesti 's distance between the 20 cattle populations used in the study. (DOCX $26 \mathrm{~kb}$ )

\section{Abbreviations}

ANG: Angus; B.C: Before Christ; BH: Brown Hanwoo; BNH: Brindle Hanwoo; BRM: Brahman; BS: Brown Swiss; DAPC: Discriminant analysis of principal components; DNA: Deoxyribonucleic acid; GIR: Gir; HF: Hereford; HGD: Hanwoo-Gaeryang-Danji; Ho: Observed heterozygosity; HOL: Holstein; Hs: Expected heterozygosity; JB: Jeju black; JER: Jersey; KPN: Korea proven bull number; LMS: Limousine; MAF: Minor allele frequency; MDS: Multi-dimensional scaling; ND: N'Dama; NEL: Nelore; ng/ul: Nanogram Per microlitre; PCA: Principal components analysis; SHK: Sheko; SNP: Single nucleotide polymorphism; YB: Yanbian.

\section{Acknowledgements}

Not applicable.

\section{Funding}

This study was supported by 2016 Postdoctoral Fellowship Program of National Institute of Animal Science and "Cooperative Research Program for Agriculture Science \& Technology Development (Project No. PJ01022002)" of Rural Development Administration, Republic of Korea. Funding body had no role in the design of the study, in the collection of the data, in analysis, in interpretation of data and in writing of the manuscript

\section{Availability of data and materials}

Data available from the Dryad Digital Repository: http://dx.doi.org/10.5061/ dryad.55f3s. The zipped folder contains cattle genotype data in plink format (Ped and map files).

\section{Authors' contributions}

AS, YC and SHL designed the study. YC, DL, BHC, HHC and SHL collected the samples and carried out the genotyping. AS analyzed the data. AS wrote the manuscript. YC and SHL provided a critical review of the manuscript. All the authors read and approved the final manuscript.

\section{Competing interests}

The authors declare that they have no competing interests.

Consent for publication

Not applicable

\section{Ethics approval and consent to participate}

No ethics statement was required for the collection of DNA samples. DNA was extracted from blood samples obtained from different veterinary practitioners across the country with the permission of the owners. The blood samples were collected for routine veterinary procedures and not explicitly for the purpose of this study.

\section{Author details}

${ }^{1}$ Division of Animal Genetics and Bioinformatics, National Institute of Animal Science, RDA, 1500, Kongjwipatjwi-ro, Iseo-myeon, Wanju-gun, Jeollabuk-do 55365, Republic of Korea. 'Division of Animal and Dairy Science, Chugnam National University, 99 Daehak-ro, Yuseong-gu, Daejeon 34134, Republic of Korea.

Received: 8 May 2016 Accepted: 30 September 2016

Published online: 20 October 2016

\section{References}

1. Payne WJA, Hodges J. Tropical cattle, origins, breeds and breeding polices. Oxford: Blackwell Science Ltd; 1997.

2. McTavish EJ, Decker JE, Schnabel RD, Taylor JF, Hillis DM. New World cattle show ancestry from multiple independent domestication events. Proc Natl Acad Sci. 2013;110:E1398-406.

3. Mannen H, Kohno M, Nagata Y, Tsuji S, Bradley D, Yeo J, et al. Independent mitochondrial origin and historical genetic differentiation in North Eastern Asian cattle. Mol Phylogenet Evol. 2004;32:539-44.

4. Choi TJ, Lee SS, Cho KH, Yoon D, Kim CD, Hwang IH, et al. Genetic diversity of traditional Korean cattle breeds based on microsatellite polymorphisms. J Agric Life Sci. 2015;49:37-46.

5. Choi JW, Choi BH, Lee SH, Lee SS, Kim HC, Yu D, et al. Whole-genome resequencing analysis of Hanwoo and Yanbian cattle to identify genomewide SNPs and signatures of selection. Mol Cell. 2015;38:466-73.

6. Kim K, Kwak W, Sung S-S, Cho S, Kim H, Yoon D, et al. A novel genetic variant database for Korean native cattle (Hanwoo): HanwooGDB. Genes Genomics. 2015:37:15-22.

7. Lee SH, Park BH, Sharma A, Dang CG, Lee SS, Choi TJ, et al. Hanwoo cattle: origin, domestication, breeding strategies and genomic selection. J Anim Sci Technol. 2014;56:2.

8. Ben Jemaa S, Boussaha M, Ben Mehdi M, Lee JH, Lee S-H. Genome-wide insights into population structure and genetic history of tunisian local cattle using the illumina bovinesnp50 beadchip. BMC Genomics. 2015;16:677.

9. Edea Z, Dadi H, Kim SW, Dessie T, Lee T, Kim H, et al. Genetic diversity, population structure and relationships in indigenous cattle populations of Ethiopia and Korean Hanwoo breeds using SNP markers. Front Genet. 2013:4:35.

10. Decker JE, McKay SD, Rolf MM, Kim J, Molina Alcalá A, Sonstegard TS, et al. Worldwide patterns of ancestry, divergence, and admixture in domesticated cattle. PLoS Genet. 2014;10:e1004254.

11. Decker JE, McKay SD, Rolf MM, Kim J, Molina Alcalá A, Sonstegard TS, et al. Data from: Worldwide patterns of ancestry, divergence, and admixture in domesticated cattle. Dryad Digital Repository; 2014. http://dx.doi.org/10.5061/ dryad.th092.

12. Browning SR, Browning BL. Rapid and accurate haplotype phasing and missing-data inference for whole-genome association studies by use of localized haplotype clustering. Am J Hum Genet. 2007;81:1084-97.

13. Purcell $S$, Neale B, Todd-Brown $K$, Thomas L, Ferreira MAR, Bender D, et al. PLINK: a tool set for whole-genome association and population-based linkage analyses. Am J Hum Genet. 2007:81:559-75.

14. Goudet J. Hierfstat, a package for $r$ to compute and test hierarchical Fstatistics. Mol Ecol Notes. 2005:5:184-6.

15. Kamvar ZN, Tabima JF, Grünwald NJ. Poppr: an R package for genetic analysis of populations with clonal, partially clonal, and/or sexual reproduction. PeerJ. 2014;2:e281

16. Jombart T. adegenet: a R package for the multivariate analysis of genetic markers. Bioinformatics. 2008:24:1403-5.

17. Alexander DH, Novembre J, Lange K. Fast model-based estimation of ancestry in unrelated individuals. Genome Res. 2009:19:1655-64.

18. Pickrell JK, Pritchard JK. Inference of population splits and mixtures from genome-wide allele frequency data. PLoS Genetics. 2012:8:e1002967.

19. Lim D, Strucken EM, Choi BH, Chai HH, Cho YM, Jang GW, et al. Genomic footprints in selected and unselected beef cattle breeds in Korea. PLoS ONE. 2016;11:e0151324 
20. Kim KS, Yeo JS, Choi CB. Genetic diversity of north-east Asian cattle based on microsatellite data. Anim Genet. 2002;33:201-4.

21. Strucken EM, Lee SH, Jang GW, Porto-Neto LR, Gondro C. Towards breed formation by island model divergence in Korean cattle. BMC Evol Bio. 2015;15:284

22. Choi JW, Liao X, Stothard P, Chung W-H, Jeon H-J, Miller SP, et al. Wholegenome analyses of Korean native and Holstein cattle breeds by massively parallel sequencing. PLoS ONE. 2014;9:e101127.

Submit your next manuscript to BioMed Central and we will help you at every step:

- We accept pre-submission inquiries

- Our selector tool helps you to find the most relevant journal

- We provide round the clock customer support

- Convenient online submission

- Thorough peer review

- Inclusion in PubMed and all major indexing services

- Maximum visibility for your research

Submit your manuscript at www.biomedcentral.com/submit 